\title{
RELATION OF MAGNETIC FIELDS WITH SOLAR ACTIVITY INDEXES IN A DESCENDING PHASE OF SOLAR CYCLE
}

\author{
Choe, Won Chol \\ Pyongyang Astronomical Observatory, Academy of Sciences, \\ Democratic People's Republic of Korea
}

\begin{abstract}
We examine the solar activity indexes (relative intensity of sunspots and faculae) of an active region \#83137 during 1983 June 21 26 . The results are compared with daily variation of magnetic fields of the region to find a characteristic of solar activity variation in the descending phase of solar cycle.
\end{abstract}

\section{INTRODUCTION}

Solar activity is related to the birth and evolution of individual active regions. The study of individual active regions in this context has been done with differential method by many researchers (e.g., Choe 1985, Bachmann 1978 , Bumba 1982). In the last solar cycle, Zirin and Werner (1967) made an extensive study of solar activities including both the photosphere, chromosphere, and upper atmosphere. By such efforts, it becomes more clear how the solar activity indexes of sunspots and prominences are related to the occurrence of flares. In this paper, we examine daily variation of solar activity indexes and magnetic field of a selected active region to find a characteristic of active region evolution in a descending phase of solar cycle.

\section{OBSERVATIONAL DATA AND ANALYSIS}

The source was an active region \# 83137 which occurred in descending phase of the 21st solar cycle. The source location was the N20 E65 on 1983 June 19. The observational data of this active region are gathered from various sources such as (1) Sunspot Data of Pyongyang Astronomical Observatory, (2) Quarterly Bulletin of Solar Activity, (3) Monthly Solar Activity of Yunnam Observatory, (4) Solnechnuie Dannuie, and (5) solar magnetic field data of Postdam in Germany.

Based on these data, we examine relative intensity and isomagnetic field lines of this region each day during $1983 \mathrm{June} 21-26$. The results for the relative intensity of sunspot and faculae are shown in Figure 1, where the solid and the dashed lines represent results for $p$-spot and $f$-spots, respectively. Magnetic field strengths on each day are also shown for comparison. In Figure 2, the variation of sunspot area is compared with that of magnetic field strength. 


\section{RESULT AND DISCUSSION}

\section{(1) Variation of Magnetic Field Strength}

On 1983 June 19 , the type of this sunspot group was B with $p$-spot in $\mathrm{N}$ polarity and $f$-spot in S-polarity. On 1983 June 21, maximum field strength of $p$-spot was $650 \mathrm{G}$ and that of $f$-spot was $320 \mathrm{G}$. On 1983 June 23 , the field strength of $p$-spot increased to $1280 \mathrm{G}$. After transmitting the central solar meridian, the magnetic field decreased gradually to reach the same value at the time of birth, on 1983 June 26. At this time, the active region was in Type D.

\section{(2) Variation of Relative Intensity of Sunspots and Faculae}

Figure 1 shows the variation of relative intensity of sunspot and faculae as well as the magnetic field evolution mentioned above. For $p$-spot, the relative intensity of sunspot decreases as field strength increases, which implies that increase of magnetic field strength is associated with the formation of sunspot group. In contrast, the relative intensity of faculae increases as field strength increases, with a certain delay. Later it decreases as field strength decreases. The behavior of $f$-spot is quite different from that of $p$-spot.

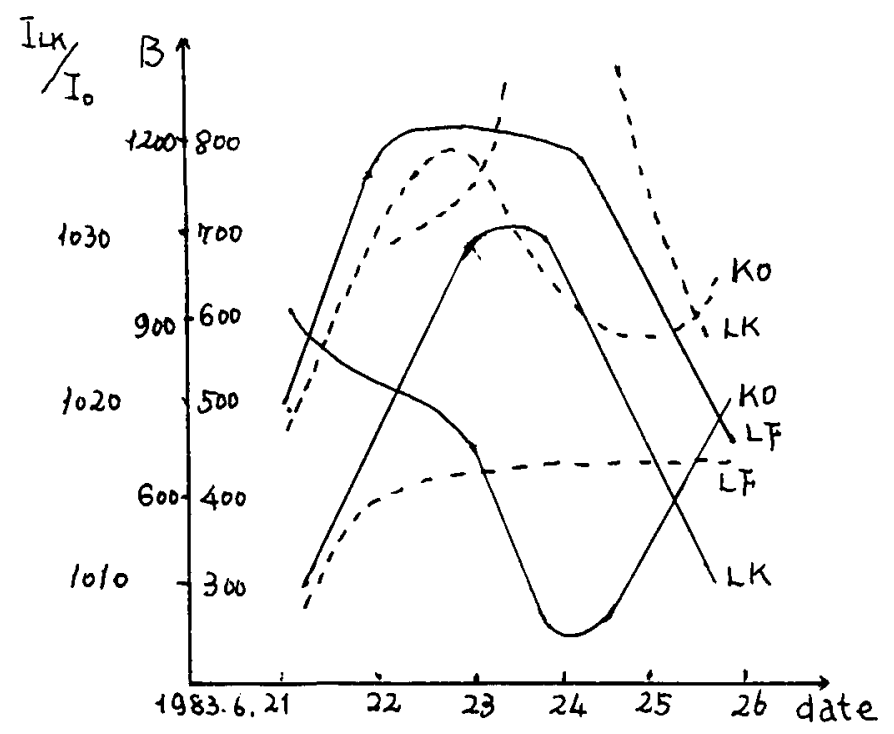

Fig.1. Variation of magnetic field strength, relative strength of sunspot and faculae with days. The solid lines represents $p$-sunspot and the dashed lines, $f$-spot. $\mathrm{LF}, \mathrm{LK}$, and $\mathrm{KO}$ denote the magnetic field strength, relative strength of faculae and relative strength of sunspot, respectively. 


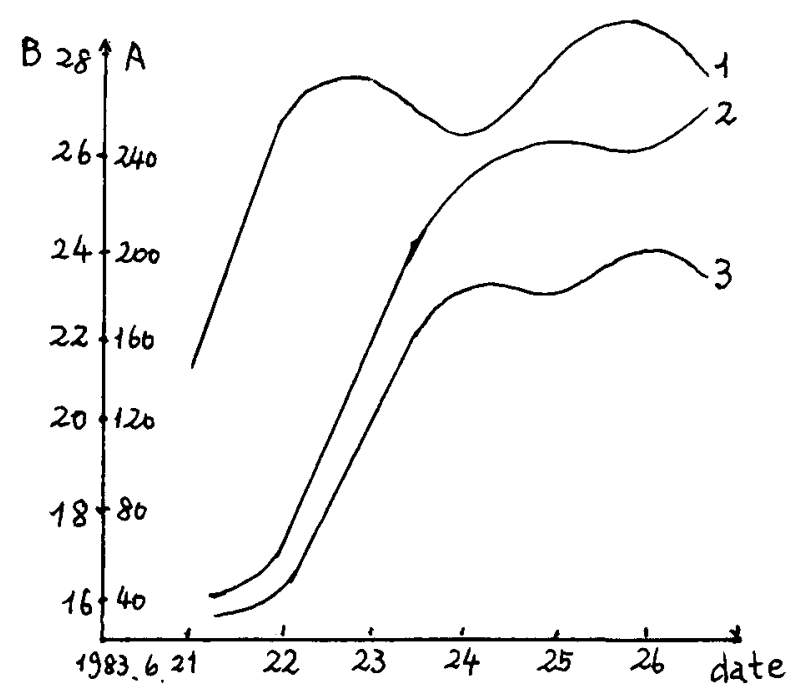

Fig. 2. Variation of area and magnetic field strength. Curves 1,2 , and 3 represent the daily variation of magnetic fields, total area of the sunspot group, and the area of main sunspot, respectively.

\section{(3) Variation of Sunspot Area}

We compare the variation of sunspot area with that of magnetic fields in Figure 2. It appears that variation of sunspot area closely follows that of magnetic fields, but with one day delay. We believe it suggests that the birth and evolution of sunspot is closely related to the magnetic field variation. In this period of observation, there were total 246 flares over the whole disk while the number of flare of this active region was 62 . It is therefore concluded that the active region was a stable one as it occurred in the descending phase.

\section{REFERENCES}

Choe, Won Chol 1985, Bull. of Astronomy, D. P. $R$ of Korea, 7, 78

Choe, Won Chol 1989, in 'Solar photosphere, Structure, convection. Magnetic fields', IAU Sympo. \# 138, Kiev Abstractbook 128

Bachmann, G. 1987, B. A. C., 29, 180

Bumba, V. et al., 1982, B. A. C., 33, 36

Zirin, H. and Werner, S. 1967, Solar phys., 1, 66 